\title{
Analysis and Improvement of Radial Spin-Echo Diffusion-Weighted Images
}

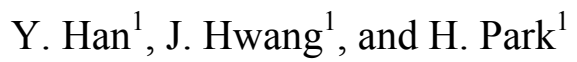 \\ ${ }^{1}$ Department of Electrical Engineering, Korea Advanced Institute of Science and Technology, Daejeon, Korea
}

\begin{abstract}
The pulsatile motion artifacts in the radial spin-echo diffusion-weighted-imaging (rSE-DWI) are analyzed and a method to improve the image quality of the rSE-DWI using the projection data regeneration method is proposed in this work. In general, ECG-triggering is used together with the rSE-DWI in order to prevent the loss of projection data, where the amount of loss depends on the data acquisition timing in the cardiac cycle. The ECG-triggering reduces the artifacts related to the cardiac pulse to some extent, but still leaves some degraded projection data while increasing the data acquisition time. Thus, the projection data regeneration method is proposed in order to improve the image quality. In the proposed method, the projection data is acquired by the rSE-DWI from which a temporary image is generated by an inverse Radon transform (IRT). The projection data acquired at each view angle is tested to figure out whether it is damaged by the pulsatile motion, and then the degraded data are replaced with the data that are regenerated by the Radon transform (RT) of the temporary image. The regeneration process is repeated until the image gains reasonable quality. In the meanwhile, the thermal noise of the signal is removed using the mask generated by thresholding the intensity of the projection data. In order to demonstrate the efficiency, the proposed method for the improvement of image quality is applied to a phantom and in-vivo images. The quality of the DW images acquired by either ECG-triggered or non-triggered rSE-DWI can be improved using the proposed method without increasing the imaging time.
\end{abstract}

Keywords-radial spin-echo diffusion-weighted images (rSE-DWI), ECG-triggering, Projection data regeneration.

\section{INTRODUCTION}

Diffusion weighted imaging (DWI) is a recent imaging technique widely used to analyze the microscopic structures and the physiological information of the organs, especially the human brain, with the help of application techniques of diffusion tensor mapping and fiber tractography [1-4].

The spin-echo diffusion-weighted echo-planar-imaging (SE-DW-EPI) sequence $[5,6]$ has won popularity because it enables a fast imaging while being fairly immune to macroscopic motions. However, it is also associated with serious image artifacts due to a magnetic susceptibility, and also with limited resolution due to the $\mathrm{T}^{*}{ }_{2}$ decay

Thus, a radial spin-echo diffusion-weighted imaging (rSE-DWI) sequence is proposed, which is insensitive to bulk motion and enables a high-resolution imaging. Although rSE-DWI is not as competitive as SE-DW-EPI in terms of the imaging time, it has many advantages, such as a slight increase in the signal-to-noise ratio as well as a reduced sensitivity to phase errors $[7,8]$, because images are reconstructed using the projection reconstruction algorithm based on the Radon transform (RT). Generally, rSE-DWI provides high-resolution images with a better image quality than SE-DW-EPI [9], but some of the projection data can be degraded due to the brain pulsatile motion, especially when high diffusion gradients are applied.

In this study, a method that improves the quality of high resolution rSE-DW images using projection regeneration method is proposed.

\section{Materials AND Methods}

The cardiac gated rSE-DWI sequence was implemented on a 3.0T MRI system manufactured by ISOL technology, Korea. A birdcage RF head coil was used for both the RF pulse transmission and the signal detection. Diffusion gradients were used with the following values: $\delta=28 \mathrm{~ms}$, $\Delta=54.8 \mathrm{~ms}$ and $\mathrm{G}=1.4 \mathrm{G} / \mathrm{cm}$. The gradients produced a diffusion value $b$ of $1000 \mathrm{~s} / \mathrm{mm}^{2}$ and images were acquired with a slice thickness of $4 \mathrm{~mm}$ using TR/TE $=2000 / 100 \mathrm{~ms} .90$ projections were acquired for the analysis of the ECGtriggering effect, and 180 projections for the application of the proposed method. 256 points per each projection were acquired for both cases with a FOV of $220 \times 220 \mathrm{~mm} 2$. For the ECG-triggering, MP150, an EMG system manufactured by Biopac System, inc. was utilized. Without ECGtriggering, the acquisition time for one set of images, containing up to 25 axial slices, was 6 min, whereas it increased to $8.5 \mathrm{~min}$ or more when ECG-triggering is used.

\section{A. Analysis}

In the case of the radial imaging, the artifacts caused by phase errors could be avoided, as the data acquired by radial imaging could be reconstructed into images using a filtered backprojection algorithm. However, there are other sources of artifacts, such as pulsatile brain motion, that result in degraded projection data, specifically the data after the 1D Fourier transform in rSE-DWI [9]. 
ECG-triggering is utilized in order to prevent the loss of projection data due to pulsatile motion, by acquiring the data during the quiet portions of the cardiac cycle [10]. However, the effect of ECG-triggering in the case of radial imaging is not analyzed in detail; thus, the projection data acquired at different stages of a cardiac cycle is analyzed in order to validate the effect of the ECG-triggering.

The first set of data was acquired at 100 to $700 \mathrm{~ms}$ after the R-peaks of a cardiac cycle from similar axial positions in two different subjects using ECG-triggering. The series of time delays after the R-peaks covers nearly the whole period of one cardiac cycle. As illustrated in Fig. 1, the data acquired at 300 400ms after the R-peak shows the fewest number of degraded projections, where dark vertical lines show the degraded projection data. When the acquisition time is synchronized with the R-peak, the data is severely damaged. However, the effect of the ECG-triggering is subject-dependent, as shown in the top and bottom rows of Fig. 1.
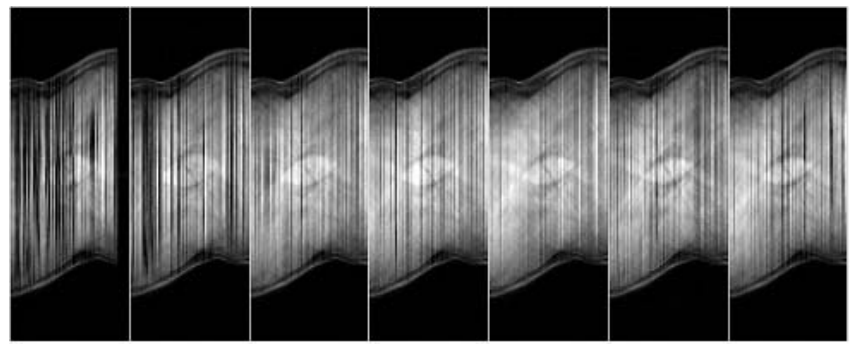

(a)

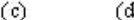

(f)

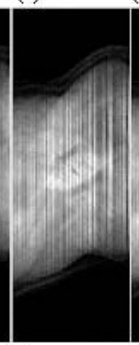

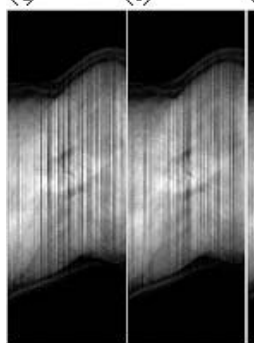

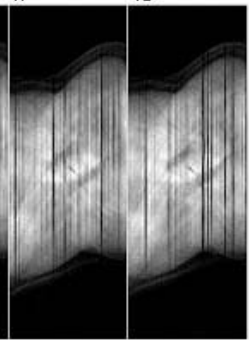

(m)

(n)

Fig.1 Projection data acquired from two subjects (top row and bottom row) with ECG-triggering after different delays from the R-peak of the cardiac cycle. The delays are (a)(h) $100 \mathrm{~ms}$, (b)(i) $200 \mathrm{~ms}$, (c)(j) $300 \mathrm{~ms}$, (d)(k) $400 \mathrm{~ms}$, (e)(1) $500 \mathrm{~ms}$, (f)(m) $600 \mathrm{~ms}$, and (g)(n) $700 \mathrm{~ms}$.

The second set of images shows different axial slices of the same subject acquired at different delays after the Rpeaks. From the images shown in Fig. 2, it can be concluded that the effectiveness of the ECG-triggering differs according to the imaging slice position, even in the same subject.

More significantly, it should be noted that ECGtriggering reduces the artifacts related to the cardiac pulse to some extent, but nonetheless leaves some degraded lines. On top of the fact that ECG-triggering is not a perfect solu- tion, the increase of acquisition time is an additional obstacle preventing the wider use of ECG-triggering. Thus, a correction algorithm is proposed in order to improve the image quality regardless of whether ECG-triggering is utilized or not.
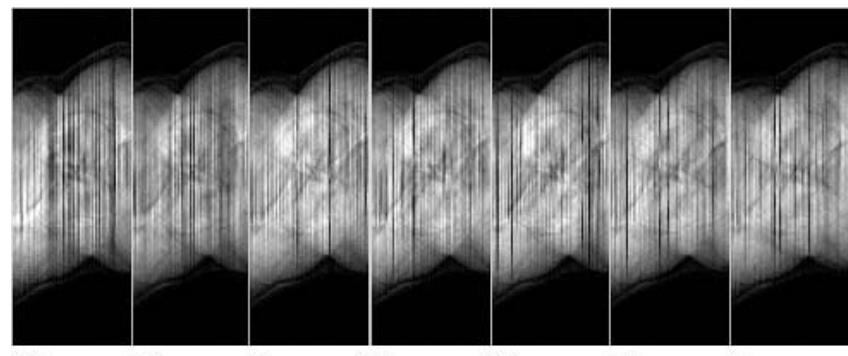
(b) (c) (d) (e) (f) (g)
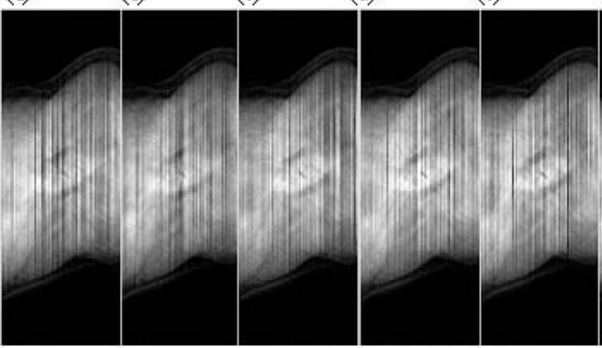

(h) (i) (j) (k) (1) (mi) 1

Fig.2 Projection data acquired from two different slices from one subject (top row and bottom row) with ECG-triggering after different delays from the R-peak of the cardiac cycle. The delays are (a)(h) $100 \mathrm{~ms}$, (b)(i) $200 \mathrm{~ms}$, (c)(j) $300 \mathrm{~ms}$, (d)(k) $400 \mathrm{~ms}$, (e)(l) $500 \mathrm{~ms}$, (f)(m) $600 \mathrm{~ms}$, and (g)(n) $700 \mathrm{~ms}$.

\section{B. Projection Data Regeneration}

As a first step, the raw data acquired by the rSE-DWI sequence is transformed to the projection data by 1D Fourier transform (FT). The projection data includes degraded data caused by pulsatile brain motions. In conventional reconstruction methods, filtered backprojection reconstruction, i.e. inverse Radon transform (IRT), is used in order to produce the final image. However, this image is used as a temporary image to generate a regenerated data. Thus, the sinogram is regenerated with the same number of projection views as in the raw data using the Radon transform (RT). At the same time, the boundaries of the original projection data are detected by thresholding the intensity to generate a mask.

To improve the image quality, only the degraded projection data of the original data is replaced with the regenerated projection data. In order to figure out the degraded projection data, the average intensity of the projection data is compared with the threshold that was empirically determined to be 0.7 times the average intensity of the entire data set. If the probabilities of the degraded and non-degraded projections are equal, the optimal threshold is the average itself. However, usually less than $30 \%$ of the projections are 
degraded (in the authors' experience) and the threshold should thus be adjusted, and is 0.7 in this case. Then, the projection data can be reconstructed to an image using IRT.

\section{RESULTS}

In order to demonstrate statistical efficiency, the proposed method was applied to the Shepp-Logan phantom image first. In vivo experiments were then performed in four healthy volunteers in order to analyze the effectiveness of the ECG-triggering and to demonstrate the feasibility of the proposed method.

\section{A. Phantom Studies}

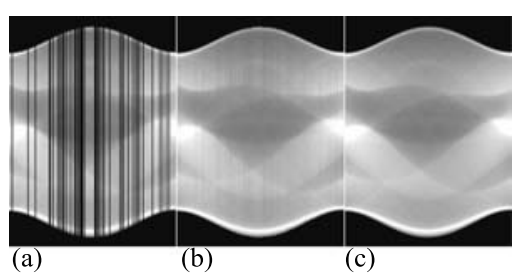

Fig.3 Sinograms of the Shepp-Logan phantom. (a) the projection data with degraded lines, (b) the restored projection data, and (c) the original projection data.
A sinogram was generated with 180 projection views from the phantom using the RT, as shown in Fig. 3(a), with some degraded projections. Using the proposed method, the projection data was regenerated, as shown in
Fig. 3(b). Figure 3(c) shows the actual projection data acquired directly from the Shepp-Logan phantom without degradation.

Each set of projection data is reconstructed to an image using the IRT. Figures 4(a) and 4(b) show images from the
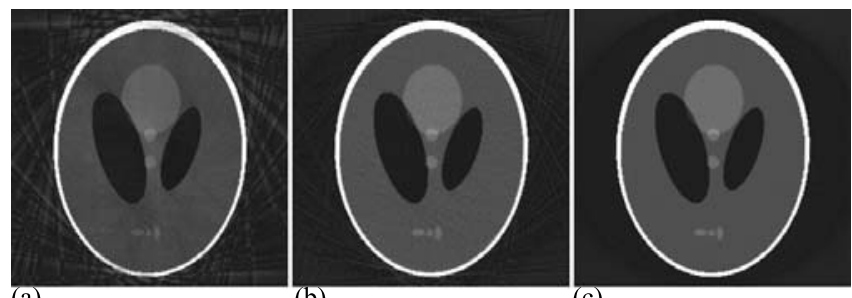

(a)

(b)

(c)
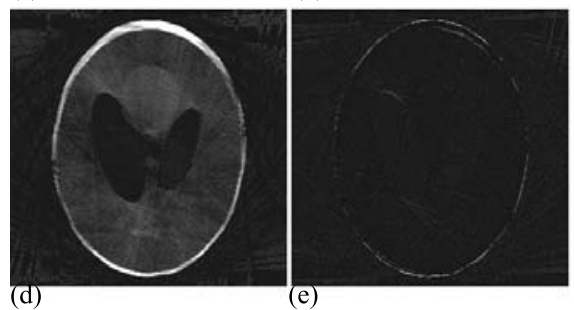

Fig.4 Shepp-Logan phantom images. (a) the image reconstructed from the degraded projection data, (b) the image reconstructed using the proposed method, (c) the original Shepp-Logan phantom image, (d) the difference image (magnified by three) of (a) and (c), and (e) the difference image (magnified by three) of (b) and (c).degraded projections and the corrected projections, respectively, and 4(c) shows the original phantom image. The difference images (Figs. 4(d), (e)) show that the image produced by the proposed method is more similar to the original image than the degraded image.

\section{B. In-vivo Studies}

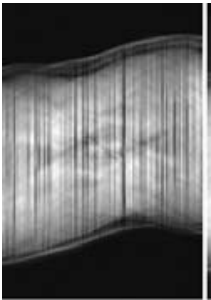

(a)

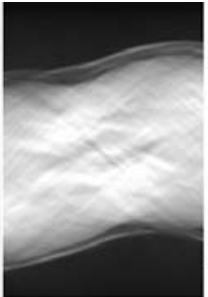

(b)

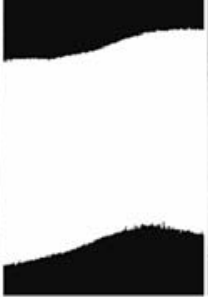

(c)

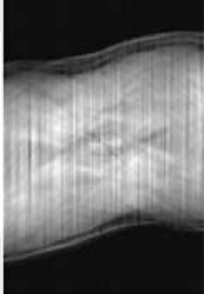

(d)
Fig. 5 Regeneration of the projection data. (a) degraded projection data, (b) mask, (c) regenerated projection data using the IRT and RT of the original data, and (d) the restored projection data

Projection data was acquired with different triggering times ranging from 100 to $700 \mathrm{~ms}$ after the R-peak of the cardiac cycle, and the proposed method was utilized to replace the degraded projection data.

From a degraded projection data (Fig. 5(a)), the projection data was regenerated by RT, as shown in Fig. 5(b). A mask, shown in Fig. 5(c), was also produced, and the degraded projection data were replaced with the regenerated projection data to produce the final projection data, as shown in Fig. 5(d).

Multislice images were then generated as shown in Fig. 6.

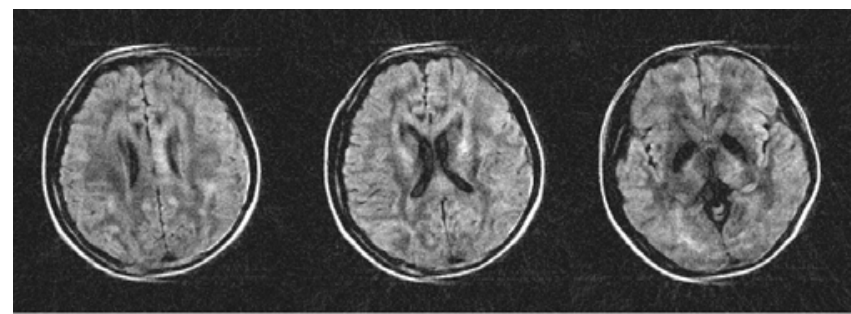

(a)

(b)

(c)

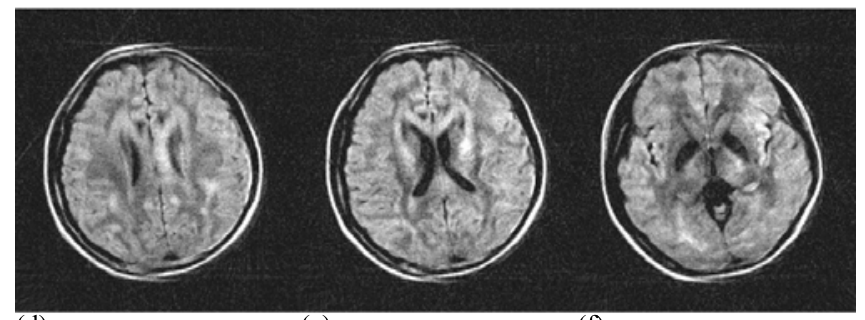

(d)

(e)

(f)

Fig.6 ultislice images before (top row) and after (bottom row) restoration of the projection data. The projection data is acquired at (a) high, (b) middle, and (c) low slices of the brain with a delay of $400 \mathrm{~ms}$ after the Rpeaks of the cardiac cycle. 
Figure 6 (top row) shows that the amount of image distortion is different according to the slice position. This means that ECG-triggering alone is not sufficient for acquiring the whole set of image slices with good image quality. Using the proposed method, these images are improved as shown in the bottom row. The images become more homogeneous, and streak artifacts are removed while preserving the details Finally, multislice images were acquired without ECGtriggering to demonstrate that the proposed method is also efficient for non-triggered rSE-DWI. Some of the images reconstructed from the projection data before and after the correction are shown in Fig.7 (top row) and Fig.7 (bottom row), respectively. They illustrate that the images can be improved using the proposed projection data regeneration method.

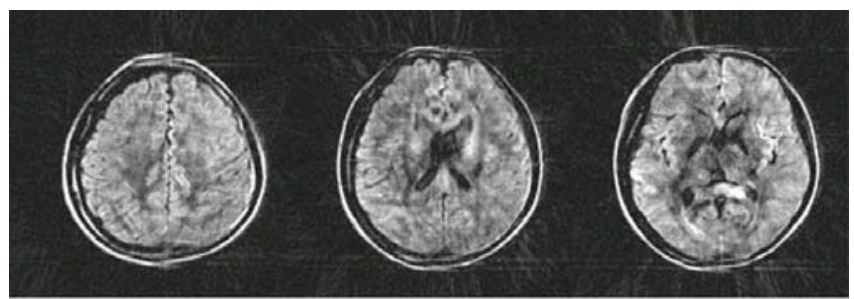

(a)

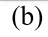

(c)

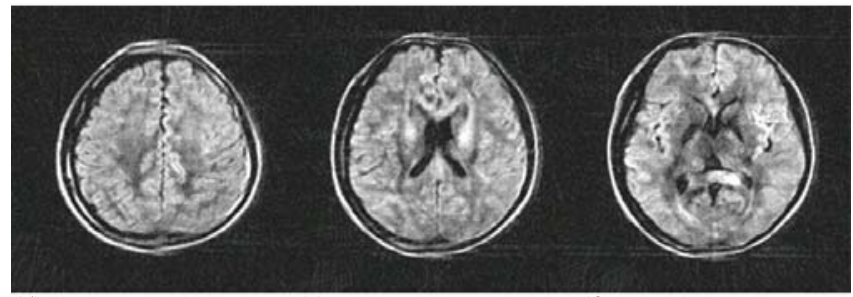

(d)

(e)

(f)

Fig.7Images reconstructed before ((a), (b), (c))) and after ((d), (e), (f)) restoration of the corresponding projection data in Fig. 8.

\section{DisCUSSION}

Despite the general improvement in the quality of the data, the rSE-DWI sequence has problems contending with the brain pulsatile motion. The fact that molecular movement due to brain pulsatile motions is much greater than the movements caused by diffusion results in degraded data in the rSE-DWI sequence, and the degradation due to the motion can be detected by inspecting the intensity. Thus, the degraded data can be replaced by regenerated projection data, as proposed in this study.

ECG-triggering increases the imaging time, as the sequence essentially waits until the trigger signal is on. Moreover, it is shown in this study that the projection data acquired using the ECG-triggering was imperfect. The image can be improved by acquiring the degraded projection data again until the projection data is $100 \%$ perfect, as Seifert et al. (9) suggested; however, this improves the image quality at the cost of the acquisition time.

Thus, a projection data regeneration method is proposed that utilizes IRT and RT consecutively. The regeneration method based on back-projection reconstruction effectively uses the features of the projection data. As a result, the proposed method improves the image quality without altering the imaging sequence.

\section{ACKNOWLEDGMENT}

This work was supported by the 10023921-2005-32, the Ministry of Commerse, Industry and Energy, Korea.

\section{REFERENCES}

1. Basser PJ, Pierpaoli C. Microstructural and physiological features of tissues elucidated by quantitative-diffusion-tensor MRI. J Magn Reson 1996;111(3): 209-219.

2. Basser PJ, Pajevic S, Pierpaoli C, Duda J, Aldroubi A. In vivo fiber tractography using DT-MNRI data. Magn Reson Med 2000;44(4):625-32.

3. Mori S, van Zijl PCM. Fiber tracking: principles and strategies - a technical review. NMR Biomed 2002;15(708):468-480.

4. Pierpaoli C, Jezzrad P, Basser PJ, Barnett A, Di Chiro G. Diffusion tensor MR imaging of the human brain. Radiology 1996; 201:637-648.

5. Turner R, Le Bihan D. Single shot diffusion imaging at 2.0 Tesla. J Magn Reson 1990;86:445-452.

6. Warach S, Gaa F, Siewert B, Wielopolski P, Edelman RR. Acute human stroke studied by whole brain echoplanar diffusion-weighted magnetic resonance imaging. Ann Neurol 1995;37:231-241.

7. Glover GH, Pauly JM. Projection Reconstructin techniques for reduction of motion effects in MRI. Magn Reson Med 1992;28:275289. 9.

8. Welch EB, Rossman PJ, Felmlee JP, Manduca A. Self-Navigated Motion Correction Using Moments of Spatial Projections in Radial MRI. Magn Reson Med 2004;52:337-345.

9. Seifert MH, Jakob PM, Jellus V, Haase A, Hillenbrand C. "HighResolution Diffusion Imaging Using a Radial Turbo-Spin-Echo Sequence: Implementation, Eddy Current Compensation, and SelfNavigation. J Magn Reson 2000;144:243-254.

10. Brockstedt S, Borg M, Geijer B, Wirestam R, Thomsen C, Holtas S, Stahlberg F. Triggering in Quantitative Diffusion Imaging with Single-Shot EPI. Acta Radiologica 1999;40:263-269.

Address of the corresponding author:

Author: Park, HyunWook, Ph.D.

Institute: Department of Electrical Engineering, Korea Advanced Institute of Science and Technology

Street: 373-1 Guseong-dong, Yuseong-gu

City: Daejeon

Country: Korea

Email: hwpark@athena.kaist.ac.kr 